\title{
Postoperative nonsteroidal antiinflammatory drugs and the prevention of heterotopic ossification after cervical arthroplasty: analysis using CT and a minimum 2-year follow-up
}

\author{
Tsung-Hsi Tu, MD, 1,3,5 Jau-Ching Wu, MD, PhD, 1,3 Wen-Cheng Huang, MD, PhD, 1,3 \\ Hsuan-Kan Chang, MD, ${ }^{1,3}$ Chin-Chu Ko, MD, , ${ }^{1,3}$ Li-Yu Fay, MD, ${ }^{1,3,4}$ Ching-Lan Wu, MD, , ${ }^{2,3}$ and \\ Henrich Cheng, MD, PhD'1,3
}

1Department of Neurosurgery, Neurological Institute, and ${ }^{2}$ Department of Radiology, Taipei Veterans General Hospital; ${ }^{3}$ School of Medicine, and ${ }^{4}$ Institute of Pharmacology, National Yang-Ming University; and ${ }^{5}$ Molecular Medicine Program, Taiwan International Graduate Program, Academia Sinica, Taipei, Taiwan

\begin{abstract}
OBJECT Heterotopic ossification ( $\mathrm{HO}$ ) after cervical arthroplasty is not uncommon and may cause immobility of the disc. To prevent $\mathrm{HO}$ formation, study protocols of clinical trials for cervical arthroplasty undertaken by the US FDA included perioperative use of nonsteroidal antiinflammatory drugs (NSAIDs). However, there are few data supporting the use of NSAIDs to prevent $\mathrm{HO}$ after cervical arthroplasty. Therefore, this study aimed to evaluate the efficacy of NSAIDs in $\mathrm{HO}$ formation and clinical outcomes.
\end{abstract}

METHODS Consecutive patients who underwent 1- or 2-level cervical arthroplasty with a minimum follow-up of 24 months were retrospectively reviewed. All patients were grouped into 1 of 2 groups, an NSAID group (those patients who had used NSAIDs postoperatively) and a non-NSAID group (those patients who had not used NSAIDs postoperatively). The formation of $\mathrm{HO}$ was detected and classified using CT in every patient. The incidence of $\mathrm{HO}$ formation, disc mobility, and clinical outcomes, including visual analog scale (VAS) scores of neck and arm pain, neck disability index (NDI) scores, and complications were compared between the two groups. Furthermore, a subgroup analysis of the patients in the NSAID group, comparing the selective cyclooxygenase (COX)-2 to nonselective COX-2 NSAID users, was also conducted for each of the above-mentioned parameters.

RESULTS A total of 75 patients (mean age $[ \pm$ SD] $46.71 \pm 9.94$ years) with 107 operated levels were analyzed. The mean follow-up duration was $38.71 \pm 9.55$ months. There were no significant differences in age, sex, and levels of arthroplasty between the NSAID and non-NSAID groups. There was a nonsignificantly lower rate of HO formation in the NSAID group than the non-NSAID group ( $47.2 \%$ vs. $68.2 \%$, respectively; $p=0.129)$. During follow-up, most of the arthroplasty levels remained mobile, with similar rates of immobile discs in the NSAID and non-NSAID groups (13.2\% and $22.7 \%$, respectively; $p=0.318$ ). Furthermore, there was a nonsignificantly lower rate of $\mathrm{HO}$ formation in the selective COX-2 group than the nonselective COX-2 group (30.8\% vs $52.5 \%$, respectively; $p=0.213$ ). The clinical outcomes, including VAS neck, VAS arm, and NDI scores at 24 months postoperatively, were all similar in the NSAID and non-NSAID groups, as well as the selective and nonselective COX-2 groups (all $p>0.05$ ).

CONCLUSIONS In this study there was a trend toward less $\mathrm{HO}$ formation and fewer immobile discs in patients who used postoperative NSAIDs after cervical arthroplasty than those who did not, but this trend did not reach statistical significance. Patients who used selective COX-2 NSAIDs had nonsignificantly less $\mathrm{HO}$ than those who used nonselective COX-2 NSAIDs. The clinical outcomes were not affected by the use of NSAIDs or the kinds of NSAIDs used (selective vs nonselective COX-2). However, the study was limited by the number of patients included, and the efficacy of NSAIDs in the prevention of $\mathrm{HO}$ after cervical arthroplasty may need further investigation to confirm these results.

http://thejns.org/doi/abs/10.3171/2014.10.SPINE14333

KEY WORDS cervical arthroplasty; NSAID; heterotopic ossification; COX-2

ABBREVIATIONS COX = cyclooxygenase; $\mathrm{HO}$ = heterotopic ossification; NDI = neck disability index; NSAID = nonsteroidal antiinflammatory drug; OPLL = ossification of the posterior longitudinal ligament; ROM = range of motion; VAS = visual analog scale.

SUBMITTED March 30, 2014. ACCEPTED October 14, 2014.

INCLUDE WHEN CITING Published online February 27, 2015; DOI: 10.3171/2014.10.SPINE14333.

DISCLOSURE The authors report no conflict of interest concerning the materials or methods used in this study or the findings specified in this paper. 
$\mathrm{A}$ NTERIOR cervical discectomy and fusion is a commonly accepted surgical procedure for cervical radiculopathy and/or myelopathy caused by disc herniation or spondylosis., ${ }^{4,11,12}$ Cervical arthroplasty has been an emerging option for similar indications with the theoretical advantage of preserving motion and reducing adjacent-segment degeneration. The continual reports of 2- to 5-year results from several prospective randomized control trials of cervical arthroplasty have demonstrated excellent clinical results similar to those from anterior cervical discectomy and fusion. 5, 15,25,26,30 However, whether cervical arthroplasty can provide protection against adjacent-segment degeneration remains debatable and needs more investigation to corroborate.

Heterotopic ossification (HO) after cervical arthroplasty was first reported by Parkinson and Sekhon in 2005. ${ }^{28}$ This ectopic bone formation could limit segmental mobility and cause unintended fusion of the index level. ${ }^{19,22,33}$ Interestingly, the reported incidence rates of $\mathrm{HO}$ after cervical arthroplasty in the literature were widely variable, ranging from $0 \%$ to $66.2 \% .^{19,22,34,36,40,41}$ During the followup evaluations most of the HOs were asymptomatic, did not affect segmental mobility, and required no reoperations., ${ }^{9,40-42}$ Nevertheless, a number of strategies were proposed to decrease $\mathrm{HO}$, including copious irrigation during the milling process, avoidance of overdistraction, and optimal carpentry of the arthroplasty. ${ }^{35}$

Nonsteroidal antiinflammatory drugs (NSAIDs) have been used as the standard of care to decrease ectopic ossification after large joint arthroplasty and spinal cord injury. ${ }^{1,2,10}$ In many of the prospective randomized controlled trials by the US FDA for cervical arthroplasty, part of their protocol was to use NSAIDs for the prevention of $\mathrm{HO}, 5,7,15,25,26,36$ Using plain radiographs for screening postoperatively, the rate of $\mathrm{HO}$ appeared to be very low in these FDA trials. On the other hand, the rates of $\mathrm{HO}$ demonstrated in other retrospective studies were remarkably higher, despite having similarly used NSAIDs for prophylaxis..$^{34,40-42}$ It is not clear if the discrepancy was caused by the method of determination for $\mathrm{HO}$, the indications of arthroplasty, or patients' compliance with the prescriptions. This study aimed to investigate the protective effect of NSAIDs for HO after cervical arthroplasty. The formation of HO was detected by CT and the mobility of each artificial disc was measured for comparison between patients who had taken NSAIDs and those who did not.

\section{Methods}

Consecutive patients who underwent 1- or 2-level cervical arthroplasty with a Bryan disc (Medtronic) at Taipei Veterans General Hospital in Taiwan from October 2006 to March 2009 were retrospectively reviewed. This study was approved by the local institutional ethics committee.

\section{Management Protocol}

Surgical indications included cervical radiculopathy and/or myelopathy caused by 1- or 2-level degenerative disc disease or spondylosis. After radiographs and CT scans, patients with the following conditions were not considered candidates for cervical arthroplasty: loss of segmental mobility due to advanced spondylosis or facet degeneration, segmental instability at the index level (> $3 \mathrm{~mm}$ translational instability or $>15^{\circ}$ angular motion), greater than $50 \%$ collapse of the normal disc height, ossification of the posterior longitudinal ligament (OPLL), and diffuse idiopathic skeletal hyperostosis. Reports have demonstrated higher incidences of OPLL in Eastern Asian countries including, Japan and Taiwan. ${ }^{39,43}$ The patients with OPLL were excluded from the current study by preoperative CT scans even if the OPLL was of the segmental type. Patients with malignancy, infection, metabolic bone disease, severe systemic disease, or traumatic ligamentous injury were also excluded.

Generous decompression of neural elements by resection of bilateral uncovertebral joints and the posterior longitudinal ligament has been routinely performed before implanting an artificial disc. The endplate preparation and drilling procedures were performed under copious irrigation with normal saline to remove bone dust. Strict hemostasis was routinely achieved intraoperatively. A drainage catheter was routinely placed prior to wound closure to prevent hematoma formation.

Postoperative NSAIDs were routinely prescribed for 2 weeks if not otherwise contraindicated by allergy or other systemic diseases. The selection of NSAIDs was at the surgeons' discretion based on clinical judgments including pain scores and medical comorbidities (e.g., history of peptic ulcers, renal function, or cardiovascular risks). The prescription dosage and frequency of each kind of NSAID was based on its pharmaceutical instruction, generally equivalent to the defined daily dose. All patients were encouraged to begin early ambulation after cervical arthroplasty.

\section{Clinical and Radiological Evaluations}

Data were collected prospectively and reviewed retrospectively. Clinical outcomes, including visual analog scale (VAS) and neck disability index (NDI) scores, were assessed within 5 days prior to the surgery and at 3, 6, 12, and 24 months postoperatively by 2 assistant specialists under supervision of the physicians. Standard anteriorposterior, lateral, and flexion-extension radiographs were obtained at the same time points postoperatively. Segmental ROM at the index level was measured with quantitative image analysis software (SmartIris, Taiwan Electronic Data Processing Co.). Cervical CT scans were acquired at follow-up more than 12 months after surgery to identify and grade the $\mathrm{HO}$ based on the classification proposed by McAfee et al. ${ }^{21}$ Radiological interpretations of segmental ROM and HO formation were made by independent radiologists and 2 neurosurgeons (T.H.T. and J.C.W.) who were blinded to both NSAID and non-NSAID groups. Immobilization of the prosthesis was defined by less than $3^{\circ}$ of motion on flexion-extension lateral radiographs.

\section{Identification of Study Group}

A chart review for postoperative NSAID use was performed. The patients were stratified into two groups: those who were administered postoperative NSAIDs (NSAID group) and those who were not (non-NSAID group). The 
non-NSAID group included those who did not take any pain medication and those who had been administered postoperative narcotics other than NSAIDs. A comparison of demographics and clinical and radiological outcomes was performed between these groups. To further evaluate the different effects of cyclooxygenase [COX]-2 selective and COX-2 nonselective NSAIDs, patients in the NSAID group were further subgrouped for comparison (i.e., the COX-2 selective vs the COX-2 nonselective group).

\section{Statistical Analysis}

All statistical analyses were performed using SPSS software (SPSS Inc.) Continuous variables were analyzed using independent t-tests, whereas the categorical data were analyzed using the Fisher's exact test. A cutoff $\mathrm{p}$ value of 0.05 was considered statistically significant.

\section{Results}

Eighty-eight consecutive patients who underwent 1or 2-level cervical arthroplasty were retrospectively reviewed. Seventy-five patients $(85.2 \%)$ completed the clinical and radiological evaluations for more than 2 years and were thus analyzed. There were 13 patients who were excluded from the analysis due to failure to give informed consent, inadequate evaluations, or lost to follow-up.

A total of 75 patients with 107 arthroplasty levels were evaluated. There were 51 males (68\%) and 24 females (32\%), with a mean age of $46.71 \pm 9.94$ years at the time of surgery. Forty-three patients $(57.3 \%)$ had 1-level surgery and 32 patients $(42.7 \%)$ had 2-level surgery. The mean clinical and radiological follow-up durations were $38.71 \pm$ 9.55 and $26.33 \pm 7.20$ months, respectively. Among the 43 patients treated by 1-level arthroplasty, $5(11.6 \%)$ were at C3-4, 6 (14.0\%) were at C4-5, 30 (69.8\%) were at C5-6, and $2(4.7 \%)$ were at C6-7. Among the 32 patients treated by 2-level surgery, $1(3.1 \%)$ was at the C3-4 and C5-6 levels, $2(6.3 \%)$ at C3-5, $19(59.4 \%)$ at C4-6, and $10(31.3 \%)$ at C5-6 (Fig. 1).

Both thin-slice CT scans and radiographs were used to evaluate the formation of HO. Among the 75 patients analyzed, $40(53.33 \%)$ had $\mathrm{HO}$ formation of various degrees, which were confirmed and classified by thin-slice CT scans (Figs. 2 and 3).

\section{NSAID and Non-NSAID Groups}

There were 53 patients (70.7\%) in the NSAID group and 22 patients $(29.3 \%)$ in the non-NSAID group (Table 1). There were no significant differences between the two groups in age, sex distribution, and number of levels that underwent arthroplasty. The clinical outcomes, including VAS scores for neck and arm pain and NDI scores preoperatively and at 24-months follow-up, were not significantly different between the two groups. There was a lower rate of $\mathrm{HO}$ formation in the NSAID group than the nonNSAID group, but it did not reach statistical significance (47.2\% vs $68.2 \%$, respectively; $p=0.129)$. Similarly, a low percentage of patients in both the NSAID and non-NSAID groups had loss of mobility $\left(<3^{\circ} \mathrm{ROM}\right.$ on the dynamic lateral radiographs) at the level that received arthroplasty (13.2\% vs $22.7 \%$, respectively; $\mathrm{p}=0.318)$.

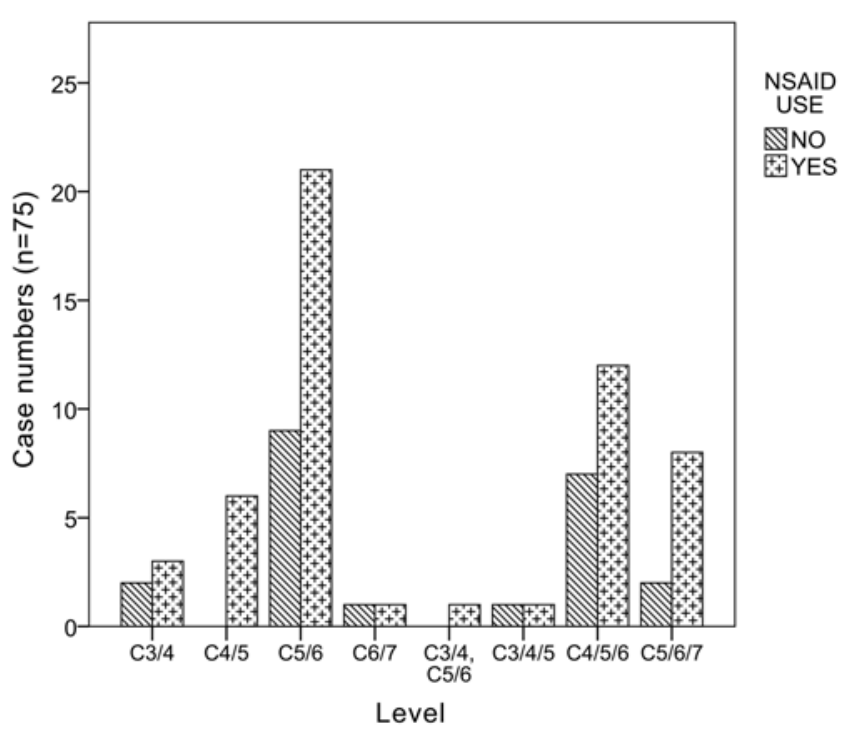

FIG. 1. Bar graph showing the distribution of cervical levels that underwent cervical arthroplasty.

\section{Selective and Nonselective COX-2 Inhibitors}

To further evaluate the effects of using different types of NSAIDs (selective and nonselective COX-2 inhibitors) for cervical arthroplasty, patients in the NSAID group were further divided into two groups: the selective COX2 and the nonselective COX-2 groups. There were 13 patients in the COX-2 group and 40 patients in the nonselective COX-2 group.

There were no significant differences in age, sex, and arthroplasty levels between these two groups (Table 2). Also, the clinical outcomes (VAS arm and neck scores, and NDI scores) were not significantly different between the two groups preoperatively and 24 -months postoperatively. However, there was a nonsignificantly lower rate of $\mathrm{HO}$ formation in the selective COX-2 group compared with the nonselective COX-2 group (30.8\% vs $52.5 \%$, respectively; $p=0.213$ ). There were similarly low rates of patients who had immobile arthroplasty in the selective compared with the nonselective COX-2 groups $(15.4 \%$ vs $12.5 \%, \mathrm{p}=1.000)$. The types of NSAIDs, including selective COX-2 and nonselective COX-2 inhibitors, are listed in Table 3.

\section{Complications}

There was no neurological deterioration or any major complications in the series. Two patients had transient hoarseness with spontaneous recovery at 6 months and 12 months postoperatively. Another 2 patients experienced intraoperative CSF leakage without clinical symptoms or wound complications. A case of C-5 palsy recovered spontaneously at 3 months postoperatively. There was no need for any reoperations during the follow-up period in the series. There were no medical complications related to the use of NSAIDs, including gastrointestinal bleeding, renal function impairment, or liver function impairment.

\section{Discussion}

This study, for the first time, specifically addressed the 

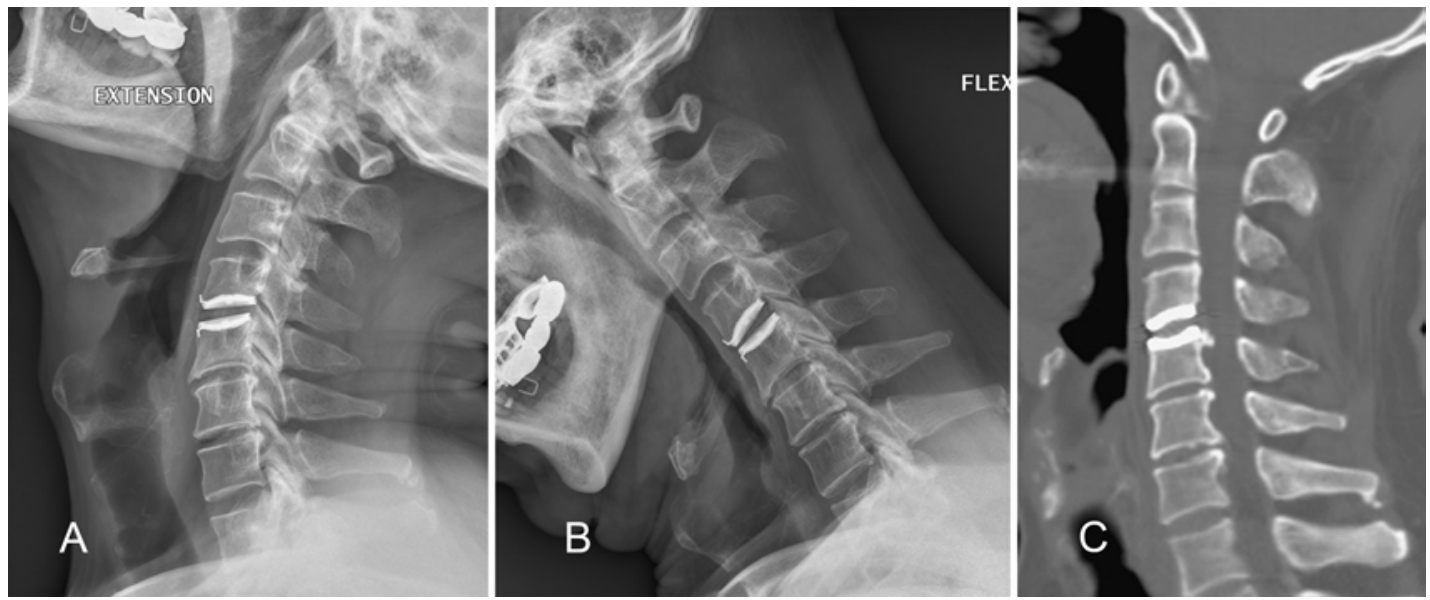

FIG. 2. Lateral radiographs (A and B) and sagittal CT scan (C) obtained 24 months postoperatively in a 66 -year-old man who underwent C4-5 cervical arthroplasty with a Bryan disc and postoperatively used an NSAID. There was HO formation behind the inferior shell of the Bryan disc.

effect of postoperative NSAIDs in cervical arthroplasty. Moreover, we also investigated the different types of NSAIDs, for example, the selective and nonselective COX2 inhibitors. The present study analyzed a cohort of 88 consecutive patients who underwent 1- or 2-level Bryan disc arthroplasty for symptomatic cervical disc degeneration or spondylosis. The follow-up rate was $85.2 \%$ with a mean duration of more than 3 years (38.71 months). More than two-thirds of the patients enrolled in this study had used NSAIDs $(n=53$ in the NSAID group and $n=22$ in the non-NSAID group). Interestingly, the rate of $\mathrm{HO}$ formation was not statistically different between the two groups. The common theory that NSAIDs adversely affect the process of bone healing could not be confirmed in this study. Moreover, there were no significant differences between selective and nonselective COX-2 inhibitors. All patients had similar neurological success and improvement in all the clinical outcome parameters, including VAS neck, VAS arm, and NDI scores. These arthroplasty patients, despite using or not using NSAIDs, or the kinds of NSAID used, had similar rates of $\mathrm{HO}$ formation, and the implanted artificial discs were similarly mobile during follow-up. To date, no other studies have investigated the issue of using NSAIDs to prevent HO.

There has been a theory that NSAIDs might decrease bone fusion via inhibition of COX catalyzed production of prostaglandins. It was reported that prostaglandins played a crucial role in bone healing by increasing the quantity and activity of osteoclasts to enhance bone absorption. Also, prostaglandins stimulate bone formation by enhancing the replication and differentiation of osteoblasts. . $^{1720,38}$ Therefore, NSAIDs are usually avoided in orthopedics when bone fusion is intended, for example in the clinical scenario of a long bone fracture. However, the avoidance of NSAIDs to enhance arthrodesis has not been supported by many clinical trials. Spinal arthroplasty adapted many experiences from arthroplasty for large joints in orthopedics such as the hip and knee, in which NSAIDs have been used for the prevention of HO. ${ }^{10,27}$ However, even for large joint arthroplasty, there are scant data demonstrating the actual efficacy of NSAIDs in the prevention of HO. The true mechanism of using NSAIDs to decrease HO is not clear. Furthermore, there are also different types of NSAIDs based on the types of COX inhibited in the phar-
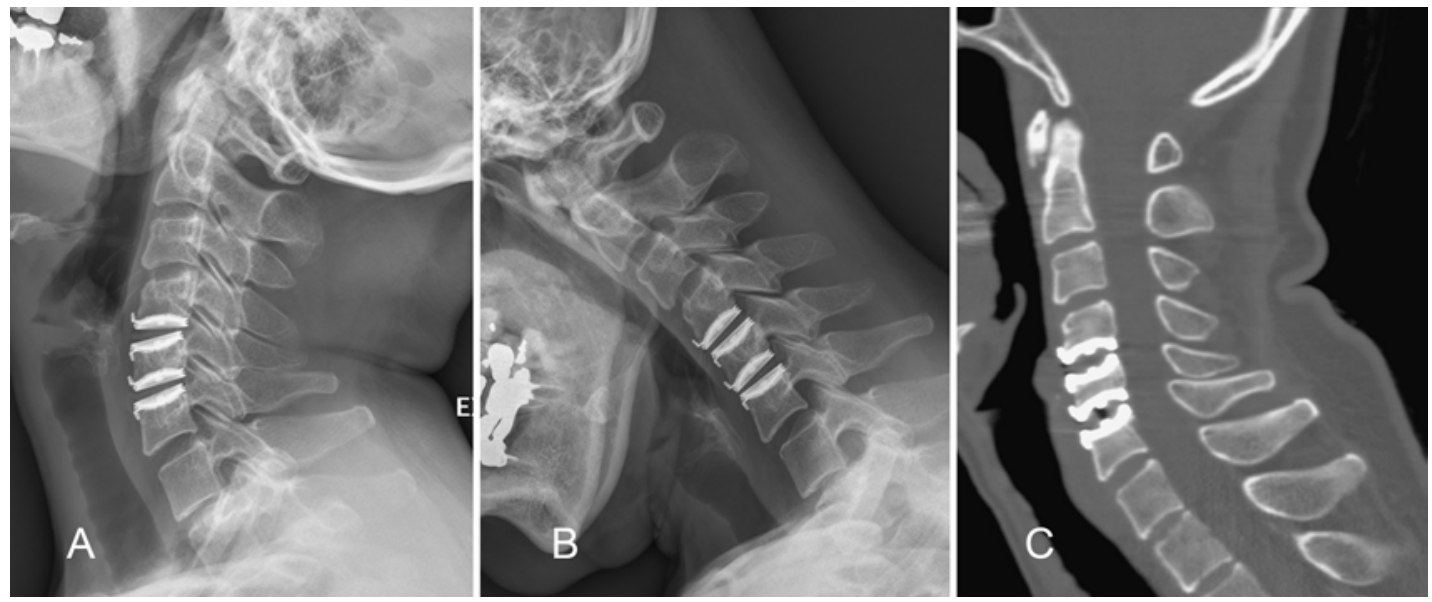

FIG. 3. Lateral radiographs (A and B) and sagittal CT scan (C) obtained 24 months postoperatively in a 46 -year-old woman who underwent C4-6 cervical arthroplasty with Bryan discs and did not use a postoperative NSAID. There was no HO formation. 
TABLE 1. Comparison of characteristics of $\mathrm{HO}$ formation in 75 patients who underwent 1- or 2-level cervical arthroplasty

\begin{tabular}{|c|c|c|c|}
\hline Variable & NSAID Use & No NSAID Use & $\mathrm{p}$ Value \\
\hline No. of patients & 53 & 22 & \\
\hline Mean age \pm SD (yrs) & $47.81 \pm 9.84$ & $44.06 \pm 9.92$ & 0.138 \\
\hline Males/females & $36 / 17$ & $15 / 7$ & 1.000 \\
\hline 1- or 2-level arthroplasty & $31 / 22$ & $12 / 10$ & 0.801 \\
\hline \multicolumn{4}{|l|}{ Mean VAS score $\pm S D$ * } \\
\hline Neck pain & $2.30 \pm 2.68$ & $2.03 \pm 2.48$ & 0.699 \\
\hline Arm pain & $1.94 \pm 2.62$ & $1.93 \pm 2.57$ & 0.984 \\
\hline Mean NDI score \pm SD $(\%)^{*}$ & $17.40 \pm 16.69$ & $18.83 \pm 15.78$ & 0.765 \\
\hline No. w/ HO formation (\%) & $25(47.2)$ & $15(68.2)$ & 0.129 \\
\hline $\begin{array}{l}\text { No. w/ immobile index level } \\
(\%) \ddagger\end{array}$ & $7(13.2)$ & $5(22.7)$ & 0.318 \\
\hline
\end{tabular}

* VAS and NDI scores were values at 24 months after the operation. $\ddagger$ Immobilization of the prosthesis was defined by less than $3^{\circ}$ of motion on flexion-extension lateral radiographs.

macological pathway. COX-1 is constitutionally expressed in normal bone, whereas COX-2 expression is stimulated during bone repair and inflammation. The antiinflammatory action of an NSAID is dependent on the inhibition of COX-2, whereas COX-1 inhibition is usually associated with unwanted effects causing interference in the regulatory and protective mechanisms. It is therefore inferred that NSAIDs affect the process of bone inflammation and healing more through inhibition of COX-2. There are some data suggesting that using both selective COX-2 inhibition and a nonselective NSAID should be done cautiously, because it could retard bone union in patients with a fracture ${ }^{37}$ Actually, selective COX-2 inhibitors may have a lower incidence of gastrointestinal bleeding, ${ }^{13,32}$ but their effect on bone healing remains uncertain..$^{23,29,31}$

There has been limited literature demonstrating the effectiveness of NSAIDs to prevent the formation of $\mathrm{HO}$ after cervical arthroplasty. The US FDA trials commonly in-

TABLE 2. Heterotopic ossification formation in the NSAID group

\begin{tabular}{|c|c|c|c|}
\hline \multirow[b]{2}{*}{ Variable } & \multicolumn{2}{|c|}{ NSAID Type } & \multirow[b]{2}{*}{ p Value } \\
\hline & $\begin{array}{l}\text { Selective } \\
\text { COX-2 }\end{array}$ & $\begin{array}{l}\text { Nonselective } \\
\text { COX-2 }\end{array}$ & \\
\hline No. of patients & 13 & 40 & \\
\hline Mean age \pm SD (yrs) & $46.79 \pm 9.38$ & $48.15 \pm 10.08$ & 0.671 \\
\hline Males/females & $8 / 5$ & $28 / 12$ & 0.734 \\
\hline 1- or 2-level arthroplasty & $8 / 5$ & $23 / 17$ & 1.000 \\
\hline \multicolumn{4}{|l|}{ VAS score $\pm S D^{*}$} \\
\hline Neck pain & $2.39 \pm 2.79$ & $2.26 \pm 2.68$ & 0.891 \\
\hline Arm pain & $1.08 \pm 2.43$ & $2.25 \pm 2.65$ & 0.169 \\
\hline Mean NDI score \pm SD $(\%)^{*}$ & $14.18 \pm 14.95$ & $18.41 \pm 17.28$ & 0.470 \\
\hline No. w/ HO formation (\%) & $4(30.8)$ & $21(52.5)$ & 0.213 \\
\hline $\begin{array}{l}\text { No. w/ immobile index level } \\
(\%) \ddagger\end{array}$ & $2(15.4)$ & $5(12.5)$ & 1.000 \\
\hline
\end{tabular}

TABLE 3. Types of NSAIDs used

\begin{tabular}{lcc}
\hline \multicolumn{1}{c}{ Group } & Dosage $(\mathrm{mg})$ & Cases \\
\hline Nonselective COX-2 NSAIDs & & \\
\hline Diclofenac & 25 & 6 \\
\hline Diclofenac & 75 & 23 \\
\hline Aceclofenac & 100 & 11 \\
\hline Total (\%) & & $40(53.3)$ \\
\hline Selective COX-2 NSAIDs & 400 & 3 \\
\hline Etodolac & 7.5 & 5 \\
\hline Meloxicam & 100 & 4 \\
\hline Nimesulide & 200 & 1 \\
\hline Celecoxib & & $13(17.3)$ \\
\hline Total (\%) & & \\
\hline Non-NSAIDs & 500 & 8 \\
\hline Acetaminophen & $65+/ 650$ & 2 \\
\hline Propoxyphene hydrochloride/acetamin- \\
$\quad$ ophen & & \\
\hline Tramadol hydrochloride/acetaminophen & $37.5+/ 325$ & 10 \\
\hline No analgesic use & & 2 \\
\hline Total (\%) & & $22(29.3)$ \\
\hline
\end{tabular}

corporated 2 weeks of postoperative NSAIDs in the management protocol. $5,25,36$ The types of NSAIDs, including selective or nonselective COX-2 types, were not specified in either of these published trials. Postoperative NSAIDs were routinely prescribed in the Prestige (Medtronic) and Bryan disc trials, but in the ProDisc-C (Synthes) study, the use of NSAIDs was left to the investigator's discretion..$^{5,15,25,26}$ The incidence rate of $\mathrm{HO}$ with osseous fusion in the Prestige, Bryan, and ProDisc-C trials was 1 of 276, 0 of 242, and 3 of 103, respectively. These rates of HO were assessed using plain radiographs. However, the true incidence of $\mathrm{HO}$ formation remains uncertain because none of these trials used CT scans for evaluation of $\mathrm{HO}$. Moreover, the reported incidence rates of $\mathrm{HO}$ formation after cervical arthroplasty varied greatly in the literature, from zero to as high as two-thirds of the patients. In a Mobi-C (LDR) study reported by Beaurain et al., with 76 cervical levels in 68 patients, HO formation was found in $67.1 \%$ of patients at the 2-year follow-up. ${ }^{3}$ Mehren et al. reported a $66.2 \% \mathrm{HO}$ occurrence rate at 1-year follow-up in a ProDisc-C series with 77 discs in 53 patients. ${ }^{22}$ They also noted significantly higher rates in multilevel cases, although the presence of $\mathrm{HO}$ did not correlate with the clinical outcomes. A $29 \%$ occurrence rate of $\mathrm{HO}$ at 2 years after cervical arthroplasty was described by Heidecke et al. in a series of 54 patients with 59 Bryan discs. Also, loss of motion was observed in $12 \%$ of the implanted artificial discs, mostly due to high-grade HO ${ }^{14}$ Previously, from our team, Tu et al. reported $\mathrm{HO}$ formation in $48.1 \%$ of treated levels with a mean patient follow-up of 2 years, using CT scans for detection and grading of HO. ${ }^{34}$ Leung et al. demonstrated a $17.8 \%$ rate of $\mathrm{HO}$ at 1-year follow-up in 90 patients who had undergone single-level disc replacement with a Bryan disc. ${ }^{19}$ The above-mentioned studies involved variable prescriptions of postoperative NSAIDs, including both duration and types. 
The causes of such a tremendous disparity in the incidence rates of $\mathrm{HO}$ formation remain elusive. The variation could be attributed to the design and material of the artificial disc, timing and modality of evaluation, surgical indications, or idiosyncrasy. Age, sex, levels of surgery, indications for surgery, and the carpentry of arthroplasty were the reported factors that could affect the formation of $\mathrm{HO}$ after cervical arthroplasty. ${ }^{6,18,24,35,36,42,44}$ In the literature, short to midterm clinical outcomes of cervical arthroplasty were not significantly impaired by $\mathrm{HO}^{34,40-42}$ There was also heterogeneity in both location and morphology of $\mathrm{HO}$ formation. ${ }^{16}$ Although the effects of different location and morphology of $\mathrm{HO}$ were not clear, it would not be surprising that those HOs that developed in the posterolateral disc space might cause nerve impingement and neural structure violation during motion. Because the current literature remains inconclusive regarding the clinical effects of $\mathrm{HO}$, the long-term consequences definitely warrant more investigation.

There are limitations as well as strengths in this study. The major limitations are the retrospective study design and insufficient period of follow-up. Thus a prospective, randomized, and controlled study with a longer time frame for follow-up (e.g., more than 5 years) could provide more information about the clinical effects of NSAIDs and the formation of HO. The number of cases was not sufficient to demonstrate statistical significance of the effect of NSAIDs in the prevention of HO. However, the clear trend implied that NSAIDs might have some effects if tested in an improved study design, such as a prospective trial. The strength of this study was in using CT scans to detect and classify the formation of HO. Under this level of scrutiny, unlike other studies that used only plain radiographs to detect $\mathrm{HO}$, higher specificity and sensitivity rates of detection for HO were anticipated. Furthermore, this is the first study to investigate the effects of NSAIDs on the formation of $\mathrm{HO}$ after cervical arthroplasty with an average follow-up of approximately 3 years. The findings may shed light on the management of patients who undergo cervical arthroplasty.

\section{Conclusions}

In this study there were trends toward less HO formation and fewer immobile discs in patients who used postoperative NSAIDs after cervical arthroplasty than those who did not, but these trends did not reach statistical significance. Patients who used selective COX-2 NSAIDs had nonsignificantly less $\mathrm{HO}$ than those who used nonselective COX-2 NSAIDs. The clinical outcomes were not affected by the use of NSAIDs or the kinds of NSAIDs used (selective vs nonselective COX-2). However, the study was limited by the number of patients included, and the efficacy of NSAIDs in the prevention of HO after cervical arthroplasty may need further investigation to confirm.

\section{References}

1. Almåsbakk K, Røysland P: Does indomethacin (IMC) prevent postoperative ectopic ossification in total hip replacement? Acta Orthop Scand 48:556, 1977

2. Banovac K, Williams JM, Patrick LD, Haniff YM: Preven- tion of heterotopic ossification after spinal cord injury with indomethacin. Spinal Cord 39:370-374, 2001

3. Beaurain J, Bernard P, Dufour T, Fuentes JM, Hovorka I, Huppert J, et al: Intermediate clinical and radiological results of cervical TDR (Mobi-C) with up to 2 years of follow-up. Eur Spine J 18:841-850, 2009

4. Bohlman HH, Emery SE, Goodfellow DB, Jones PK: Robinson anterior cervical discectomy and arthrodesis for cervical radiculopathy. Long-term follow-up of one hundred and twenty-two patients. J Bone Joint Surg Am 75:1298-1307, 1993

5. Burkus JK, Haid RW, Traynelis VC, Mummaneni PV: Longterm clinical and radiographic outcomes of cervical disc replacement with the Prestige disc: results from a prospective randomized controlled clinical trial. J Neurosurg Spine 13:308-318, 2010

6. Chung SB, Muradov JM, Lee SH, Eoh W, Kim ES: Uncovertebral hypertrophy is a significant risk factor for the occurrence of heterotopic ossification after cervical disc replacement: survivorship analysis of Bryan disc for single-level cervical arthroplasty. Acta Neurochir (Wien) 154:1017-1022, 2012

7. Coric D, Nunley PD, Guyer RD, Musante D, Carmody CN, Gordon CR, et al: Prospective, randomized, multicenter study of cervical arthroplasty: 269 patients from the Kineflex|C artificial disc investigational device exemption study with a minimum 2-year follow-up. Clinical article. J Neurosurg Spine 15:348-358, 2011

8. Emery SE, Bohlman HH, Bolesta MJ, Jones PK: Anterior cervical decompression and arthrodesis for the treatment of cervical spondylotic myelopathy. Two to seventeen-year follow-up. J Bone Joint Surg Am 80:941-951, 1998

9. Fay LY, Huang WC, Tsai TY, Wu JC, Ko CC, Tu TH, et al: Differences between arthroplasty and anterior cervical fusion in two-level cervical degenerative disc disease. Eur Spine J 23:627-634, 2014

10. Fransen M, Neal B: Non-steroidal anti-inflammatory drugs for preventing heterotopic bone formation after hip arthroplasty. Cochrane Database Syst Rev (3):CD001160, 2004

11. Fraser JF, Härtl R: Anterior approaches to fusion of the cervical spine: a metaanalysis of fusion rates. J Neurosurg Spine 6:298-303, 2007

12. Gore DR, Sepic SB: Anterior discectomy and fusion for painful cervical disc disease. A report of 50 patients with an average follow-up of 21 years. Spine (Phila Pa 1976) 23:2047-2051, 1998

13. Hawkey CJ: COX-2 inhibitors. Lancet 353:307-314, 1999

14. Heidecke V, Burkert W, Brucke M, Rainov NG: Intervertebral disc replacement for cervical degenerative disease-clinical results and functional outcome at two years in patients implanted with the Bryan cervical disc prosthesis. Acta Neurochir (Wien) 150:453-459, 2008

15. Heller JG, Sasso RC, Papadopoulos SM, Anderson PA, Fessler RG, Hacker RJ, et al: Comparison of BRYAN cervical disc arthroplasty with anterior cervical decompression and fusion: clinical and radiographic results of a randomized, controlled, clinical trial. Spine (Phila Pa 1976) 34:101-107, 2009

16. Jin YJ, Park SB, Kim MJ, Kim KJ, Kim HJ: An analysis of heterotopic ossification in cervical disc arthroplasty: a novel morphologic classification of an ossified mass. Spine $\mathbf{J}$ 13:408-420, 2013

17. Kawaguchi H, Pilbeam CC, Harrison JR, Raisz LG: The role of prostaglandins in the regulation of bone metabolism. Clin Orthop Relat Res (313):36-46, 1995

18. Kim HJ, Kelly MP, Ely CG, Dettori JR, Riew KD: The risk of adjacent-level ossification development after surgery in the cervical spine: are there factors that affect the risk? A systematic review. Spine (Phila Pa 1976) 37 (22 Suppl):S65S74, 2012 
19. Leung C, Casey AT, Goffin J, Kehr P, Liebig K, Lind B, et al: Clinical significance of heterotopic ossification in cervical disc replacement: a prospective multicenter clinical trial. Neurosurgery 57:759-763, 2005

20. Lin CH, Jee WS, Ma YF, Setterberg RB: Early effects of prostaglandin E2 on bone formation and resorption in different bone sites of rats. Bone 17 (4 Suppl):255S-259S, 1995

21. McAfee PC, Cunningham BW, Devine J, Williams E, Yu-Yahiro J: Classification of heterotopic ossification (HO) in artificial disk replacement. J Spinal Disord Tech 16:384-389, 2003

22. Mehren C, Suchomel P, Grochulla F, Barsa P, Sourkova P, Hradil J, et al: Heterotopic ossification in total cervical artificial disc replacement. Spine (Phila Pa 1976) 31:2802-2806, 2006

23. Meunier A, Aspenberg P, Good L: Celecoxib does not appear to affect prosthesis fixation in total knee replacement: a randomized study using radiostereometry in 50 patients. Acta Orthop 80:46-50, 2009

24. Mummaneni PV, Amin BY, Wu JC, Brodt ED, Dettori JR, Sasso RC: Cervical artificial disc replacement versus fusion in the cervical spine: a systematic review comparing longterm follow-up results from two FDA trials. Evid Based Spine Care J 3 (S1):59-66, 2012

25. Mummaneni PV, Burkus JK, Haid RW, Traynelis VC, Zdeblick TA: Clinical and radiographic analysis of cervical disc arthroplasty compared with allograft fusion: a randomized controlled clinical trial. J Neurosurg Spine 6:198-209, 2007

26. Murrey D, Janssen M, Delamarter R, Goldstein J, Zigler J, Tay B, et al: Results of the prospective, randomized, controlled multicenter Food and Drug Administration investigational device exemption study of the ProDisc-C total disc replacement versus anterior discectomy and fusion for the treatment of 1-level symptomatic cervical disc disease. Spine J 9:275-286, 2009

27. Neal BC, Rodgers A, Clark T, Gray H, Reid IR, Dunn L, et al: A systematic survey of 13 randomized trials of non-steroidal anti-inflammatory drugs for the prevention of heterotopic bone formation after major hip surgery. Acta Orthop Scand 71:122-128, 2000

28. Parkinson JF, Sekhon LH: Cervical arthroplasty complicated by delayed spontaneous fusion. Case report. J Neurosurg Spine 2:377-380, 2005

29. Pountos I, Georgouli T, Calori GM, Giannoudis PV: Do nonsteroidal anti-inflammatory drugs affect bone healing? A critical analysis. ScientificWorldJournal 2012:606404, 2012

30. Sasso RC, Anderson PA, Riew KD, Heller JG: Results of cervical arthroplasty compared with anterior discectomy and fusion: four-year clinical outcomes in a prospective, randomized controlled trial. J Bone Joint Surg Am 93:1684-1692, 2011

31. Seidenberg AB, An YH: Is there an inhibitory effect of COX2 inhibitors on bone healing? Pharmacol Res 50:151-156, 2004

32. Silverstein FE, Faich G, Goldstein JL, Simon LS, Pincus T, Whelton A, et al: Gastrointestinal toxicity with celecoxib vs nonsteroidal anti-inflammatory drugs for osteoarthritis and rheumatoid arthritis: the CLASS study: a randomized controlled trial. JAMA 284:1247-1255, 2000

33. Suchomel P, Jurák L, Benes V III, Brabec R, Bradác O, Elgawhary S: Clinical results and development of heterotopic ossification in total cervical disc replacement during a 4-year follow-up. Eur Spine J 19:307-315, 2010
34. Tu TH, Wu JC, Huang WC, Guo WY, Wu CL, Shih YH, et al: Heterotopic ossification after cervical total disc replacement: determination by CT and effects on clinical outcomes. J Neurosurg Spine 14:457-465, 2011

35. Tu TH, Wu JC, Huang WC, Wu CL, Ko CC, Cheng H: The effects of carpentry on heterotopic ossification and mobility in cervical arthroplasty: determination by computed tomography with a minimum 2-year follow-up. Clinical article. J Neurosurg Spine 16:601-609, 2012

36. Upadhyaya CD, Wu JC, Trost G, Haid RW, Traynelis VC, Tay B, et al: Analysis of the three United States Food and Drug Administration investigational device exemption cervical arthroplasty trials. J Neurosurg Spine 16:216-228, 2012

37. Vuolteenaho K, Moilanen T, Moilanen E: Non-steroidal antiinflammatory drugs, cyclooxygenase- 2 and the bone healing process. Basic Clin Pharmacol Toxicol 102:10-14, 2008

38. Woodiel FN, Fall PM, Raisz LG: Anabolic effects of prostaglandins in cultured fetal rat calvariae: structure-activity relations and signal transduction pathway. J Bone Miner Res 11:1249-1255, 1996

39. Wu JC, Chen YC, Liu L, Huang WC, Chen TJ, Lo SS, et al: Conservatively treated ossification of the posterior longitudinal ligament increases the risk of spinal cord injury: a nationwide cohort study. J Neurotrauma 29:462-468, 2012

40. Wu JC, Huang WC, Tsai HW, Ko CC, Fay LY, Tu TH, et al: Differences between 1- and 2-level cervical arthroplasty: more heterotopic ossification in 2-level disc replacement. Clinical article. J Neurosurg Spine 16:594-600, 2012

41. Wu JC, Huang WC, Tsai TY, Fay LY, Ko CC, Tu TH, et al: Multilevel arthroplasty for cervical spondylosis: more heterotopic ossification at 3 years of follow-up. Spine (Phila Pa 1976) 37:E1251-E1259, 2012

42. Wu JC, Huang WC, Tu TH, Tsai HW, Ko CC, Wu CL, et al: Differences between soft-disc herniation and spondylosis in cervical arthroplasty: CT-documented heterotopic ossification with minimum 2 years of follow-up. J Neurosurg Spine 16:163-171, 2012

43. Wu JC, Liu L, Chen YC, Huang WC, Chen TJ, Cheng H: Ossification of the posterior longitudinal ligament in the cervical spine: an 11-year comprehensive national epidemiology study. Neurosurg Focus 30(3):E5, 2011

44. Yi S, Shin DA, Kim KN, Choi G, Shin HC, Kim KS, et al: The predisposing factors for the heterotopic ossification after cervical artificial disc replacement. Spine J 13:1048-1054, 2013

\section{Author Contributions}

Conception and design: JC Wu, Tu, Huang, Ko, CL Wu, Cheng. Acquisition of data: Tu, Chang, CL Wu. Analysis and interpretation of data: JC Wu, Tu, Huang, Ko, Fay. Drafting the article: Tu. Critically revising the article: JC Wu, Huang, Chang, Fay, Cheng. Reviewed submitted version of manuscript: all authors. Approved the final version of the manuscript on behalf of all authors: JC Wu. Statistical analysis: Tu, Ko. Administrative/technical/material support: Huang, Cheng. Study supervision: Huang, Cheng.

\section{Correspondence}

Jau-Ching Wu, Neural Regeneration Center, Department of Neurosurgery, Neurological Institute, Taipei Veterans General Hospital, Rm. 509, 17F, No. 201, Shih-Pai Rd., Sec. 2, Peitou, Taipei 11217, Taiwan. email: jauching@gmail.com. 